\title{
Three-operator Problems in Banach Spaces
}

\author{
Jesús M.F. Castillo, Muneo Chō, Manuel González * \\ Departamento de Matemáticas, Universidad de Extremadura, E-06006 Badajoz, Spain \\ castillo@unex.es \\ Department of Mathematics, Kanagawa University, Hiratsuka 259 - 1293, Japan \\ chiyom01@kanagawa-u.ac.jp \\ Departamento de Matemáticas, Universidad de Cantabria, E-39071 Santander, Spain \\ manuel.gonzalez@unican.es
}

Received May 31, 2018

\begin{abstract}
We study the analogue of 3-space problems for classes of operators acting on Banach spaces. We show examples of classes of operators having or failing the 3-operator property, and give several methods to obtain classes with this property.

Key words: Three-space property, extending operators, lifting operators, semigroup, operator ideal.
\end{abstract}

AMS Subject Class. (2000): 46B03, 46B08, 46B10.

\section{INTRODUCTION}

The 3-space problem for a class or property $\mathcal{P}$ of Banach spaces is the question of whether a Banach space $X$ has $\mathcal{P}$ provided that a certain subspace $Y$ and the corresponding quotient space $X / Y$ have $\mathcal{P}$. If so, it is then said that $\mathcal{P}$ is a 3 -space property. For instance, reflexivity, separability or having density character $\aleph$ are 3 -space properties. Three-space problems have been a popular topic of research because a positive answer for $\mathcal{P}$ yields a technique to get spaces with $\mathcal{P}$; while a negative answer necessarily provides a new insight into Banach space constructions. Moreover, to get either positive results or counterexamples more often than not requires a blend of different techniques. The monograph [12] contains a thorough, not-too-outdated, treatment of the 3 -space problem in Banach spaces.

${ }^{*}$ The research of the first author was supported in part by Project IB16056 de la Junta de Extremadura; that of the first and third authors was supported in part by MINECO (Spain), Project MTM2016-76958. This paper benefited from a stay in 2016 of Castillo and González at Kanagawa University invited by Prof. Cho. 
In this paper we consider the analogue of 3 -space problem for operators by means of what we will call the 3-operator property (see Definition 1), which was introduced in [29]. We also consider some weak versions of the 3 -operator property that were introduced in [13]. Some of them can be enjoyed by an operator ideal $\mathcal{A}$ and maintain close connections with the fact that the space ideal of $\mathcal{A}$ enjoys the 3 -space property. We first observe that no (nontrivial) operator ideal can enjoy the 3-operator property, and so we turn our attention to other classes, most remarkably semigroups. Our main result (Theorem 1) gives a characterization of the semigroups that satisfy the 3-operator property. As a consequence we show in Corollary 1 that several semigroups considered in [13] have the 3-operator property. We also describe some categorical methods to obtain new classes with the 3-operator property from a class that have that property. Finally we consider some operator ideals related with the separably injective spaces studied in [5] that provide examples satisfying or failing some 3 -operator-like properties considered in the paper.

\section{THREE-OPERATOR PROPERTIES}

A class $\mathcal{A}$ of operators is called an operator ideal if it contains the class $\mathcal{F}$ of finite-rank operators, is closed under addition, and the composition of an element of $\mathcal{A}$ with any operator is in $\mathcal{A}$. Typical examples of operator ideals are the classes $\mathcal{L}$ of all operators, $\mathcal{K}$ of compact operators and $\mathcal{W}$ of weakly compact operators. A class $\mathcal{S}$ of operators is a semigroup if it contains the bijective operators, it is stable under composition, and given $S \in \mathcal{L}(U, X)$ and $T \in \mathcal{L}(V, Y)$,

$$
S, T \in \mathcal{S} \Longleftrightarrow S \oplus T \in \mathcal{S},
$$

where $S \oplus T: U \oplus V \rightarrow X \oplus Y$ is defined by $S \oplus T(u, v)=(S u, T v)$.

The notion of operator ideal was thoroughly studied by Pietsch [24] (see [25] for details) while the notion of semigroup was considered in [1] and [18, Chapter 6].

An operator ideal $\mathcal{A}$ has associated a class of Banach spaces $S p(\mathcal{A})=\{X$ : $\left.i d_{X} \in \mathcal{A}\right\}$, where $i d_{X}$ is the identity in $X$, which is called the space ideal of $\mathcal{A}$ [24]. Space ideals are, according to [24], classes of Banach spaces containing the finite dimensional spaces and stable under products and complemented subspaces. Observe that $X \in S p(\mathcal{A})$ if and only if $\mathcal{L}(X, X)=\mathcal{A}(X, X)$. Similarly, a semigroup $\mathcal{S}$ has associated a class of spaces $\operatorname{ker}(\mathcal{S})=\left\{X: 0_{X} \in \mathcal{S}\right\}$, and $X \in \operatorname{ker}(\mathcal{S})$ if and only if $\mathcal{L}(X)=\mathcal{S}(X)$. We will see in Proposition 1 that, for an operator ideal $\mathcal{A}, S p(\mathcal{A})$ coincides with the kernel of the semi- 
groups $\mathcal{A}_{+}$and $\mathcal{A}_{-}$associated to $\mathcal{A}$. Given a class of operators $\mathcal{A}$, we denote $\mathcal{A}^{d}=\left\{T \in \mathcal{L}: T^{*} \in \mathcal{A}\right\}$, the dual class of $\mathcal{A}$. Note that if $\mathcal{A}$ is an operator ideal or a semigroup then so does $\mathcal{A}^{d}[24$, Theorem 4.4.2], [18, Proposition 6.1.4].

The homological notation will be useful in this paper: recall that an exact sequence

$$
0 \longrightarrow Y \stackrel{i}{\longrightarrow} X \stackrel{q}{\longrightarrow} Z \longrightarrow 0
$$

of Banach spaces and (linear continuous) operators is a diagram in which the kernel of each arrow coincides with the image of the preceding one. The open mapping theorem yields that $\mathrm{Y}$ is isomorphic to a subspace of $X$ such that $X / i(Y)$ is isomorphic to $Z$. Consequently, $\mathcal{P}$ is a 3 -space property if whenever one has a short exact sequence (2.1) in which the spaces $Y, Z$ have $\mathcal{P}$ then also $X$ has $\mathcal{P}$. Still according to [24], given a space ideal $\mathbf{A}$, the class of all operators that factorize through a space in $\mathbf{A}$ form an operator ideal $O p(\mathbf{A})$. Thus, if the spaces with $\mathcal{P}$ form a space ideal (or even if not with some ad hoc amendments), $\mathcal{P}$ is a 3 -space property if given a commutative diagram

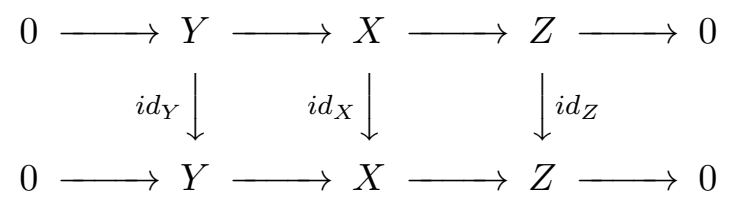

with exact rows, if $i d_{Y}, i d_{Z} \in O p(\mathcal{P})$ then also $i d_{X} \in O p(\mathcal{P})$. Consequently, the following concept makes sense.

Definition 1. A class $\mathcal{A}$ of operators is said to have the 3 -operator property if given a commutative diagram with exact rows

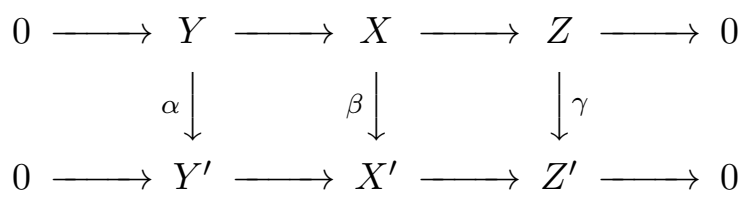

if $\alpha, \gamma \in \mathcal{A}$ then also $\beta \in \mathcal{A}$.

This notion was introduced by Zeng and Zhong in [29], where they prove that the classes of upper and lower semi-Fredholm operators satisfy it (a new proof will be given in Corollary 3), and Zeng proved in [28] that some classes of operators defined in terms of spectral properties satisfy the 3 -operator property. 
Unfortunately, $\mathcal{L}$ is the only operator ideal enjoying the 3-operator property: the diagram

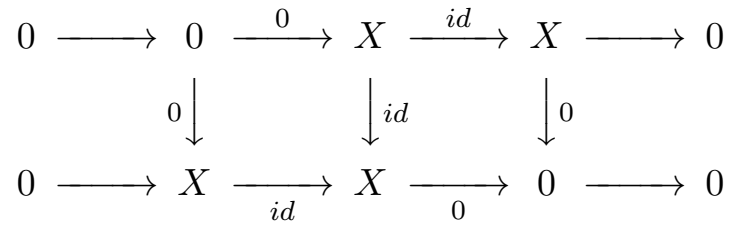

shows that an operator ideal satisfying the 3-operator property contains the identity of every Banach space. Nevertheless, classes of operators with the 3-operator property do exist:

Example 1. The classical 3-lemma from homological algebra [12, p. 3] shows that the following classes of operators have the 3-space property:

(a) The class inj of injective operators.

(b) The class dens of dense range operators.

(c) The class emb of (into) embedding operators.

(d) The class surj of surjective operators.

(e) The class iso of bijective operators.

Two "3-operator-like" properties were introduced in [13, Definitions 2 and 5 and Propositions 10 and 16].

Definition 2. Let $\mathcal{A}$ be a class of operators.

(a) We say that $\mathcal{A}$ satisfies the $3 S_{-}$property if given a push-out diagram

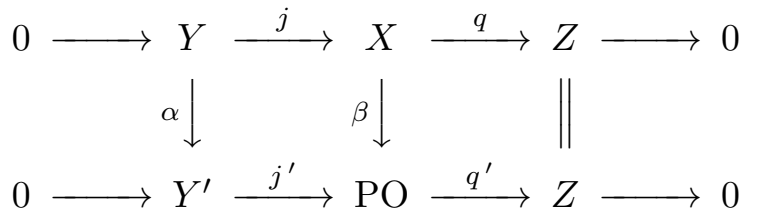

with exact rows then $\alpha, q \in \mathcal{A} \Rightarrow \beta \in \mathcal{A}$.

(b) We say that $\mathcal{A}$ satisfies the $3 S_{+}$property if given a pull-back diagram

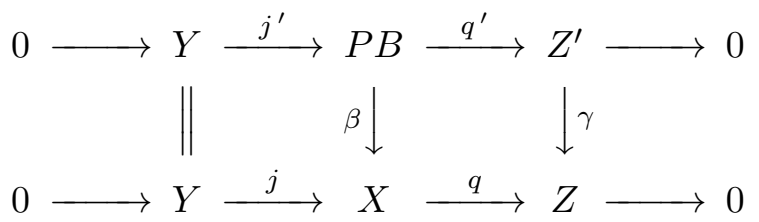

with exact rows then $\gamma, j \in \mathcal{A} \Rightarrow \beta \in \mathcal{A}$. 
While $\mathcal{L}$ is the only operator ideal that satisfies the 3-operator property, some operator ideals satisfy the $3 S_{-}$and $3 S_{+}$properties:

(a) The operator ideals of strictly singular and strictly cosingular operators enjoy respectively properties $3 S_{-}$and $3 S_{+}$.

This fact is essentially proved in [14, Proposition 3.2], and explicitly in [10, Lemma 8] and [13, Proposition 11].

(b) The operator ideal of $p$-converging operators $\mathcal{C}_{p}$ studied in [11] satisfies the $3 S_{-}$and $3 S_{+}$properties for $1 \leq p \leq \infty$ [13, Propositions 13 and 17]. Note that $\mathcal{C}_{\infty}=\mathcal{C}$, the completely continuous operators, and $\mathcal{C}_{1}=\mathcal{U}$, the unconditionally converging operators.

(c) Let $\mathcal{K} \mathcal{W}, \mathcal{R}, \mathcal{U}, \mathcal{C}$ and $\mathcal{W C}$ denote the operator ideals of compact, weakly compact, Rosenthal, unconditionally convergent, completely continuous, and weakly completely continuous operators (see [24] for their definitions), and let $\mathcal{A}$ be one of these operator ideals. Then $\mathcal{A}$ satisfies the $3 S_{+}$property and its dual $\mathcal{A}^{d}$ satisfies the $3 S_{-}$property [13, Propositions 15 and 9$]$.

The following result will be useful later:

Lemma 1 . If $\mathcal{A}$ has the property $3 S_{-}$then $\mathcal{A}^{d}$ has the property $3 S_{+}$; and similarly, if $\mathcal{A}$ has the property $3 S_{+}$then $\mathcal{A}^{d}$ has the property $3 S_{-}$.

Proof. For the proof of the first result, it is enough to observe that

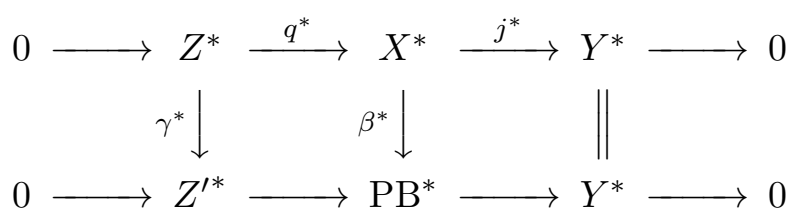

which is the conjugate of diagram (2.5), is also a push-out diagram like (2.4). Indeed, $\mathrm{PB}$ is the pull-back of $\gamma$ and $q$, and $\mathrm{PB}^{*}$ can be identified with the push-out of $\gamma^{*}$ and $q^{*}$. We refer to [13, Section 2] for the details.

The proof of the second result is similar.

Those properties are interesting for the study of 3-space problems. Indeed, as it was shown in [13, Proposition 18], if an operator ideal $\mathcal{A}$ satisfies one of the properties $3 S_{-}$or $3 S_{+}$then the space ideal $S p(\mathcal{A})$ satisfies the 3 -space property. 
For applications of the $3 S_{+}$and $3 S_{-}$properties to the analysis of commutative diagrams of operators we refer to [13, Propositions 14 and 19] and [14, Proposition 3.3].

\section{SEMigroups OF OPERATORS}

The classes of operators appearing in Example 1 satisfy the definition of semigroup presented at the beginning of Section 2. This notion of semigroup if closely related with that of operator ideal. It was proved in [1] and [18, Chapter 6] that every operator ideal $\mathcal{A}$ has associated two semigroups $\mathcal{A}_{+}$ and $\mathcal{A}_{-}$defined as follows.

Definition 3. Let $\mathcal{A}$ be an operator ideal and let $T \in \mathcal{L}(X, Y)$.

(a) $T \in \mathcal{A}_{+}$if for every $S \in \mathcal{L}(Z, X), T S \in \mathcal{A}$ implies $S \in \mathcal{A}$.

(b) $T \in \mathcal{A}_{-}$if for every $S \in \mathcal{L}(Y, Z), S T \in \mathcal{A}$ implies $S \in \mathcal{A}$.

As a direct consequence of the definitions we obtain the following equalities.

Proposition 1. For every operator ideal $\mathcal{A}, \operatorname{Sp}(\mathcal{A})=\operatorname{ker}\left(\mathcal{A}_{+}\right)=\operatorname{ker}\left(\mathcal{A}_{-}\right)$.

The following two 3-operator-like properties for semigroups were introduced in [13, Definition 1] in a slightly different way.

Definition 4. Let $\mathcal{A}$ be a class of operators. We say that:

(a) $\mathcal{A}$ satisfies the $3 \mathrm{PO}$ property if given a push-out diagram like (2.4), if $\alpha \in \mathcal{A}$ then also $\beta \in \mathcal{A}$.

(b) $\mathcal{A}$ satisfies the $3 \mathrm{~PB}$ property if given a pull-back diagram like (2.5), if $\gamma \in \mathcal{A}$ then also $\beta \in \mathcal{A}$.

As in the case of the 3-operator property, an operator ideal satisfying the $3 \mathrm{PO}$ property or the the $3 \mathrm{~PB}$ property contains the identity of every Banach space, hence it is $\mathcal{L}$. For the $3 \mathrm{PO}$ property, note that we can construct diagrams like (2.4) with $Z$ arbitrary; and we can similarly argue for the $3 \mathrm{~PB}$ property. 
Proposition 2. Let $\mathcal{A}$ be an operator ideal.

(a) If $\mathcal{A}$ is injective then $\mathcal{A}_{+}$satisfies the $3 \mathrm{~PB}$ property.

(b) If $\mathcal{A}$ is surjective then $\mathcal{A}_{-}$satisfies the $3 \mathrm{PO}$ property.

(c) If $\mathcal{A}_{+}$satisfies the $3 \mathrm{PO}$ property or $\mathcal{A}_{-}$satisfies the $3 \mathrm{~PB}$ property then $S p(\mathcal{A})$ has the 3-space property.

Proof. (a) Suppose $\mathcal{A}$ is injective and $\gamma$ in diagram (2.5) belongs to $\mathcal{A}_{+}$. We have to show that $\beta \in \mathcal{A}_{+}$.

Let $S: W \rightarrow$ PB such that $\beta S \in \mathcal{A}$. From $\gamma \in \mathcal{A}_{+}$and $q \beta S=\gamma q^{\prime} S \in \mathcal{A}$, we get $q^{\prime} S \in \mathcal{A}$. The map $J: P B \rightarrow X \oplus Z^{\prime}$ given by $J v=\left(\beta v, q^{\prime} v\right)$ is an (into) embedding, and $\beta S, q^{\prime} S \in \mathcal{A}$ implies $J S \in \mathcal{A}$; hence $S \in \mathcal{A}$ because $\mathcal{A}$ is injective.

(b) Suppose $\mathcal{A}$ is surjective and $\alpha$ in diagram (2.4) belongs to $\in \mathcal{A}_{-}$. We have to show that $\beta \in \mathcal{A}_{-}$.

Let $S: \mathrm{PO} \rightarrow W$ such that $S \beta \in \mathcal{A}$. From $\alpha \in \mathcal{A}_{-}$and $S \beta j=S j^{\prime} \alpha \in \mathcal{A}$, we get $S j^{\prime} \in \mathcal{A}$. The map $Q: Y^{\prime} \oplus X \rightarrow P O$ given by $Q\left(y^{\prime}, x\right)=j^{\prime} y^{\prime}+\beta x$ is surjective, and $S \beta, S j^{\prime} \in \mathcal{A}$ implies $S Q \in \mathcal{A}$; hence $S \in \mathcal{A}$ because $\mathcal{A}$ is surjective.

(c) See [13, Proposition 7].

Clearly the $3 \mathrm{PO}$ property implies the $3 S_{+}$, the $3 \mathrm{~PB}$ property implies the $3 S_{-}$property, and Lemma 1 has its counterpart admitting a similar proof:

Lemma 2. If $\mathcal{A}$ has the $3 \mathrm{~PB}$ property then $\mathcal{A}^{d}$ has the $3 \mathrm{PO}$ property; and similarly, if $\mathcal{A}$ has the $3 \mathrm{PO}$ property then $\mathcal{A}^{d}$ has the $3 \mathrm{~PB}$ property.

The following result is useful to understand the 3-operator property.

Theorem 1. Let $\mathcal{A}$ be a class of operators stable by composition and containing the bijective operators. Then $\mathcal{A}$ has the 3-operator property if and only if it has properties $3 \mathrm{PO}$ and $3 \mathrm{~PB}$.

Proof. The direct implication is trivial, because each identity is in $\mathcal{A}$. For the converse implication, given the commutative diagram in Definition 1

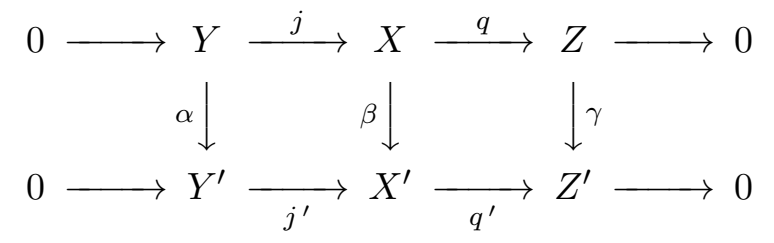


we consider the push-out diagram of $\alpha$ and $j$ :

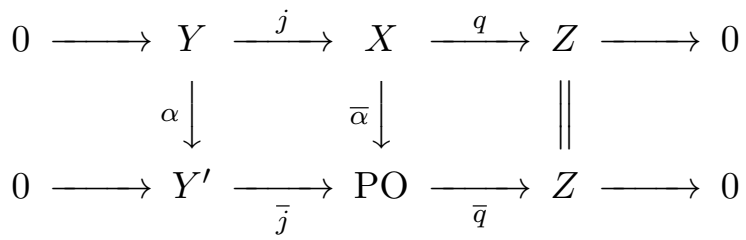

where

$$
\begin{gathered}
\mathrm{PO}=\left(Y^{\prime} \times X\right) / \Delta \quad \text { with } \quad \Delta=\{(\alpha y,-j y): y \in Y\} \\
\bar{j} y^{\prime}=\left(y^{\prime}, 0\right)+\Delta, \quad \bar{\alpha} x=(0, x)+\Delta \quad \text { and } \quad \bar{q}\left(\left(y^{\prime}, 0\right)+\Delta\right)=q x .
\end{gathered}
$$

Moreover we consider the pull-back diagram of $\gamma$ and $q^{\prime}$ :

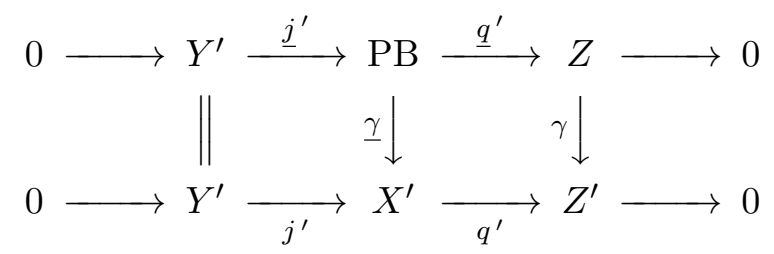

where

$$
\begin{gathered}
\mathrm{PB}=\left\{\left(x^{\prime}, z\right) \in X^{\prime} \times Z: q^{\prime} x^{\prime}=\gamma z\right\}, \\
\underline{j}^{\prime} y^{\prime}=\left(j^{\prime} y^{\prime}, 0\right), \quad \underline{\gamma}\left(x^{\prime}, z\right)=x^{\prime} \quad \text { and } \quad \underline{q}^{\prime}\left(x^{\prime}, z\right)=z .
\end{gathered}
$$

Let us show that the map $\Psi: \mathrm{PO} \rightarrow \mathrm{PB}$ defined by

$$
\Psi\left(\left(y^{\prime}, x\right)+\Delta\right)=\left(j^{\prime} y^{\prime}+\beta x, q x\right)
$$

is a bijective operator such that $\beta=\underline{\gamma} \Psi \bar{\alpha}$. We do it in several steps:

(1) $\Psi$ takes values in PB: $q^{\prime}\left(j^{\prime} y^{\prime}+\beta x\right)=q^{\prime} \beta x=\gamma q x$.

(2) $\Psi$ is well-defined since $\left(y^{\prime}, x\right) \in \Delta$ implies $y^{\prime}=\alpha y$ and $x=-j y$ for some $y \in Y$. Then $j^{\prime} y^{\prime}+\beta x=j^{\prime} \alpha y-\beta j y=0$ and $q x=-q j y=0$.

(3) $\Psi$ is bijective because the diagram

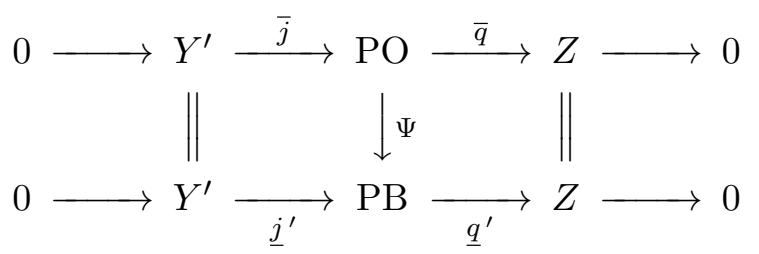


is commutative. Indeed,

$$
\begin{gathered}
\underline{q^{\prime}} \Psi\left(\left(y^{\prime}, x\right)+\Delta\right)=\underline{q}^{\prime}\left(j^{\prime} y^{\prime}+\beta x, q x\right)=q x=\bar{q}\left(\left(y^{\prime}, x\right)+\Delta\right) \\
\text { and } \Psi \bar{j} y^{\prime}=\Psi\left(\left(y^{\prime}, 0\right)+\Delta\right)=\left(j^{\prime} y^{\prime}, 0\right)=\underline{j}^{\prime} y^{\prime} .
\end{gathered}
$$

(4) For every $x \in X$,

$$
\underline{\gamma} \Psi \bar{\alpha} x=\underline{\gamma} \Psi((0, x)+\Delta)=\underline{\gamma}(\beta x, q x)=\beta x .
$$

To conclude the proof, since $\mathcal{A}$ has properties $3 \mathrm{PO}$ and $3 \mathrm{~PB}, \alpha \in \mathcal{A}$ implies $\bar{\alpha} \in \mathcal{A}$ and $\gamma \in \mathcal{A}$ implies $\underline{\gamma} \in \mathcal{A}$, hence $\beta=\underline{\gamma} \Psi \bar{\alpha} \in \mathcal{A}$.

Several examples of semigroups satisfying the $3 \mathrm{PO}$ or the $3 \mathrm{~PB}$ property were given in [13].

Proposition 3. ([13, Theorem 1]). Let $\mathcal{A}$ denote one of the operator ideals $\mathcal{K} \mathcal{W}, \mathcal{R}, \mathcal{U}, \mathcal{C}$ or $\mathcal{W C}$. Then the semigroup $\mathcal{A}_{+}$satisfies the $3 \mathrm{PO}$ property and the semigroup $\left(\mathcal{A}^{d}\right)_{-}$satisfies the $3 \mathrm{~PB}$ property.

Note that $\mathcal{K}=\mathcal{K}^{d}$ by Schauder's theorem and $\mathcal{W}=\mathcal{W}^{d}$ by Gantmacher's theorem. As a consequence,

Corollary 1. Let $\mathcal{A}$ denote one of the operator ideals $\mathcal{K} \mathcal{W}, \mathcal{R}, \mathcal{U}, \mathcal{C}$ or $\mathcal{W C}$. Then the semigroups $\mathcal{A}_{+}$and $\left(\mathcal{A}^{d}\right)_{-}$satisfy the 3-operator property.

Proof. The operator ideal $\mathcal{A}$ is injective, hence the semigroup $\mathcal{A}_{+}$satisfies the 3 PB property by Proposition 2 . Since $\mathcal{A}_{+}$also satisfies the 3 PO property (Proposition 3), it has the 3-operator property by Theorem 1.

Similarly, since $\mathcal{A}^{d}$ is surjective and $\left(\mathcal{A}^{d}\right)_{-}$satisfies the $3 \mathrm{~PB}$ property,

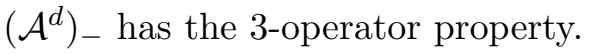

Since $\mathcal{C}_{p}$ is injective (hence $\mathcal{C}_{p}^{d}$ is surjective), $\left(\mathcal{C}_{p}\right)_{+}$satisfies the 3 PB property and $\left(\mathcal{C}_{p}^{d}\right)_{-}$the $3 \mathrm{PO}$ property. However the following questions remain open:

(a) Do the semigroups $\left(\mathcal{C}_{p}\right)_{+}$and $\left(\mathcal{C}_{p}^{d}\right)_{-}$satisfy the the 3 -operator property?

(b) Does $\left(\mathcal{C}_{p}\right)_{+}$satisfy the 3 PO property, or $\left(\mathcal{C}_{p}^{d}\right)_{-}$satisfy the $3 \mathrm{~PB}$ property?

For the obtention of lifting result for sequences when the quotient map belongs to one of the semigroups $\mathcal{W}_{+}, \mathcal{R}_{+}, \mathcal{C}_{+}$or $\mathcal{W} \mathcal{C}_{+}$we refer to [19]. 


\section{Categorical methods to obtain 3-Operator Classes}

A functor $\mathcal{F}$ acting on the category of Banach spaces is said to be exact if it transforms exact sequences into exact sequences. Examples of exact functors can be found in [12, Section 2.2] and include the following ones:

- The duality functor, $\mathcal{F}(X)=X^{*}$ and $\mathcal{F}(T)=T^{*}$ (as well as the Biduality functor, $\alpha$-transfinite dual, etc).

- The residual functor, $\mathcal{F}(X)=X^{* *} / X$ and $\mathcal{F}(T)=T^{* *} / T$, where if $T: X \rightarrow Y$ then $T^{* *} / T: X^{* *} / X \rightarrow Y^{* *} / Y$ is the operator induced by $T^{* *}$.

- The ultrapower functor, $\mathcal{F}(X)=X_{\mathfrak{U}}$ and $\mathcal{F}(T)=T_{\mathfrak{U}}$, where $\mathfrak{U}$ is a non-trivial ultrafilter.

- The ultra-residual functor, $\mathcal{F}(X)=X_{\mathfrak{U}} / X$ and $\mathcal{F}(T)=T_{\mathfrak{U}} / T$.

- The $C(K, \cdot)$ functor, $K$ a compact space: $C(K, X)$ is the Banach space of all continuous functions $f: K \rightarrow X$, and for each $T: X \rightarrow Y$, the operator $C(K, T): C(K, X) \rightarrow C(K, Y)$ is defined by $C(K, T)(f)(k)=$ $T(f(k))$.

One obviously has:

Proposition 4. Let $\mathcal{A}$ be a class of operators with the 3-operator property and let $\mathcal{F}$ be an exact functor. Then the class

$$
\mathcal{F}^{-1}(\mathcal{A})=\{T: \mathcal{F}(T) \in \mathcal{A}\}
$$

has the 3-operator property.

Let us show that nontrivial results can be obtained with this method. It was proved in [20] that $\mathcal{W}_{+}$coincides with the class of tauberian operators introduced in [22] and $\mathcal{W}_{-}$coincides with the class of cotauberian operators introduced in [27]. Since (see [18]),

$$
\mathcal{W}_{+}=\left\{T: T^{* *} / T \in \mathbf{i n j}\right\} \quad \text { and } \quad \mathcal{W}_{-}=\left\{T: T^{* *} / T \in \mathbf{d e n s}\right\}
$$

one has

Corollary 2. The semigroups $\mathcal{W}_{+}$of tauberian and $\mathcal{W}_{-}$of cotauberian operators have the 3-operator property. 
It was proved in [3] that $\left(\mathcal{W}_{+}\right)^{d d}=\left\{T: T^{* *} \in \mathcal{W}_{+}\right\}$is properly contained in $\mathcal{W}_{+}$. It follows from Proposition 4 and Corollary 2 that $\left(\mathcal{W}_{+}\right)^{d d}$ has the 3-operator property.

In [21] (see also [4]) the operators $S$ that can be represented as $T^{* *} / T$ for some operator $T$ are studied. Recall that many Banach spaces (such as separable of weakly compactly generated [8]) are linearly isometric to some $X^{* *} / X$. The operators $T$ such that $T^{* *} / T \in \mathbf{e m b}$ are called strongly tauberian in [26]. In a similar way as in the case of tauberian operators, it can be shown that the class of strongly tauberian operators has the 3-operator property.

The semigroup $\mathcal{K}_{+}$coincides with the class of upper semi-Fredholm operators (operators with closed range and finite dimensional kernel) while $\mathcal{K}_{-}$ coincides with the class of lower semi-Fredholm operators (operators with closed and finite codimensional range) (see [17]). Since

$$
\begin{aligned}
& \mathcal{K}_{+}=\left\{T: T_{\mathfrak{U}} / T \in \mathbf{i n j}\right\}=\left\{T: T_{\mathfrak{U}} / T \in \mathbf{e m b}\right\} \\
& \mathcal{K}_{-}=\left\{T: T_{\mathfrak{U}} / T \in \mathbf{d e n s}\right\}=\left\{T: T_{\mathfrak{U}} / T \in \mathbf{s u r j}\right\}
\end{aligned}
$$

one has

COROLlaRY 3. The semigroups $\mathcal{K}_{+}$and $\mathcal{K}_{-}$have the 3 -operator property.

As in Corollaries 2 and 3 , it is possible to show other classes with the 3 -operator property by applying exact functors to known classes that satisfy that property. It would be interesting to identify some of them with known classes of operators.

\section{Separable injeCtivity REVISITED}

The density character of a Banach space $X$, dens $(X)$, is the smallest cardinal $\aleph$ for which $X$ has a subset of cardinality $\aleph$ spanning a dense subspace.

Definition 5. Let $\aleph$ be a cardinal.

A Banach space $X$ is said to be $\aleph$-injective if every operator $t: Y \rightarrow X$ admits an extension $T: E \rightarrow X$ to a superspace $E \supset Y$ whenever $\operatorname{dens}(E)<$ $\aleph$.

The space $X$ is said to be universally $\aleph$-injective if every operator $t$ : $Y \rightarrow X$ admits an extension $T: E \rightarrow X$ to a superspace $E \supset Y$ whenever $\operatorname{dens}(Y)<\aleph$. 
The separably injective and universally separably injective Banach spaces (corresponding to the choice $\aleph=\aleph_{1}$ ) have been recently studied in the monograph [5]. We present now an operator approach to those ideas.

Definition 6. Let $\aleph$ be a cardinal and let $T: X \rightarrow Y$ be an operator.

(i) $T \in \mathcal{E}_{0}(\aleph)$ if for every super-space $E \supset X$ with $\operatorname{dens}(E / X)<\aleph$ there exists an extension $\widehat{T}: E \rightarrow Y$.

(ii) $T \in \mathcal{E}(\aleph)$ if for every subspace $M \subset X$ with $\operatorname{dens}(M)<\aleph$ and every superspace $E \supset M$, the restriction $\left.T\right|_{M}: M \rightarrow Y$ admits an extension $\widehat{\left.T\right|_{M}}: E \rightarrow Y$.

Proposition 5. The classes $\mathcal{E}_{0}(\aleph)$ and $\mathcal{E}(\aleph)$ are operator ideals.

Proof. It is obvious that $\mathcal{E}_{0}(\aleph)+\mathcal{E}_{0}(\aleph) \subset \mathcal{E}_{0}(\aleph), \mathcal{E}(\aleph)+\mathcal{E}(\aleph) \subset \mathcal{E}(\aleph)$, and both classes contain the finite rank operators. We prove that given $T \in$ $\mathcal{E}_{0}(\aleph)\left(X, X^{\prime}\right), R \in \mathcal{L}\left(X^{\prime}, Y^{\prime}\right)$ and $S \in \mathcal{L}(Y, X)$ one gets $R T S \in \mathcal{E}_{0}(\aleph)\left(Y, Y^{\prime}\right)$.

Let $E$ be a superspace of $Y$ such that dens $(E / Y)<\aleph$ and consider the diagram

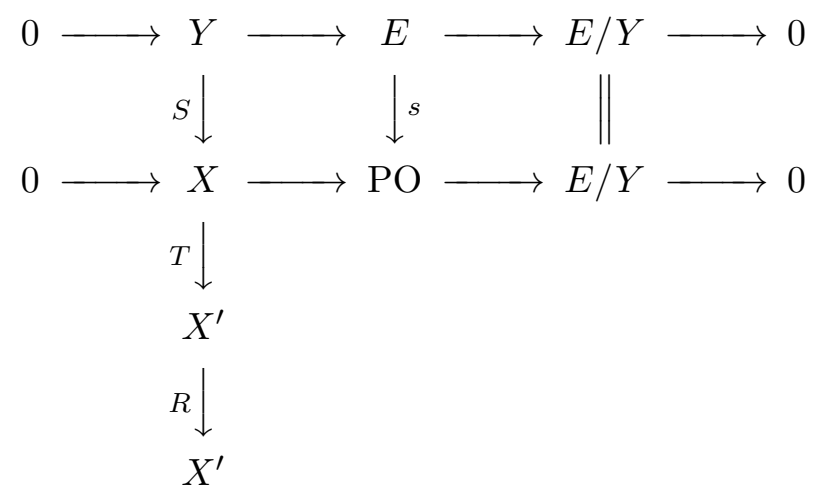

Since $T \in \mathcal{E}_{0}$, it admits an extension $t: \mathrm{PO} \rightarrow X^{\prime}$, thus the operator $R T S$ admits the extension $R t s$. The proof for $\mathcal{E}(\aleph)$ is analogous.

Our interest in these uncommon operator ideals appears explained in the next result.

Proposition 6. Let $\aleph$ be a cardinal.

(a) $X \in S p\left(\mathcal{E}_{0}(\aleph)\right)$ if and only if $X$ is $\aleph$-injective.

(b) $X \in S p(\mathcal{E}(\aleph))$ if and only if $X$ is universally $\aleph$-injective. 
Proof. It is clear that $I d_{X} \in \mathcal{E}_{0}(\aleph)$ if and only if $X$ is complemented in every superspace $E$ so that dens $(E / X)<\aleph$; namely, $X$ is $\aleph$-injective. It is also clear that if $X$ is universally $\aleph$-injective then $I d_{X} \in \mathcal{E}(\aleph)$. To prove the converse, let $Y$ be a Banach space with $\operatorname{dens}(Y)<\aleph$ which is a subspace of a space $E$, and let $t: Y \rightarrow X$ be an operator. Consider the diagram

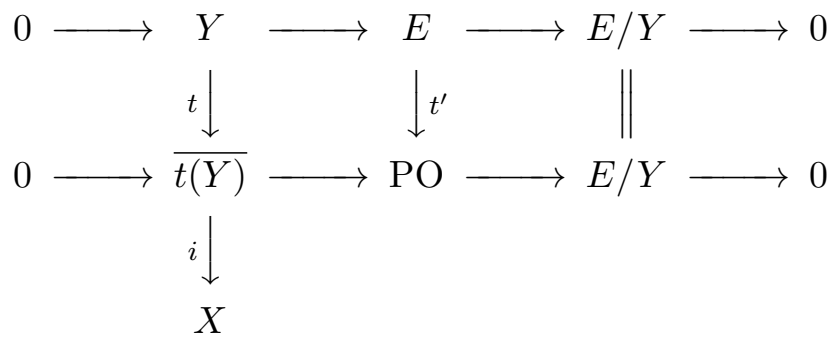

Since $\operatorname{dens}(\overline{t(Y)}) \leq \operatorname{dens}(Y)$, the canonical inclusion $i$ can be extended to PO, therefore $t$ can be extended to $E$, which shows that $X$ is universally $\aleph$-injective.

It is therefore clear that $\mathcal{E}_{0}(\aleph)($ resp. $\mathcal{E}(\aleph))$ are non-injective operator ideals containing (although probably different from) the class of all operators that factorize through an $\aleph$-injective (resp. universally $\aleph$-injective) space. They are likely not to be surjective either. More ad hoc versions of these operator ideals have been introduced and studied by Domański [16]: he fixes a Banach space $Z$ and considers the ideas $\mathcal{E}_{Z}$ of those operators $T: X \rightarrow Y$ such that for every super-space $E \supset X$ with $E / X \simeq Z$ there exists an extension $\widehat{T}: E \rightarrow Y$. Therefore, it will turn out (see [5] for details) that $\mathcal{E}_{0}(\aleph)=\bigcup_{Z} \mathcal{E}_{Z}$ when the intersection runs over all spaces $Z$ with density character $<\aleph$. One has

Proposition 7. The class $\mathcal{E}_{0}(\aleph)$ satisfies the $3 S_{-}$property.

Proof. We consider a push-out diagram

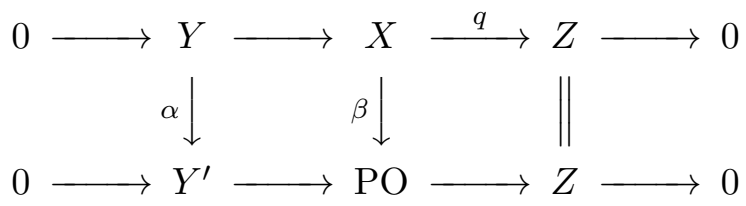

with $q, \alpha \in \mathcal{E}_{0}(\aleph)$, and we have to show that $\beta \in \mathcal{E}_{0}(\aleph)$. Let $X^{\prime}$ be a superspace of $X$ so that dens $\left(X^{\prime} / X\right)<\aleph$. Our goal is to extend $\beta$ to an operator $B: X^{\prime} \rightarrow$ PO. 
Let $Q: X^{\prime} \rightarrow Z$ be an extension of $q$. The commutative diagram

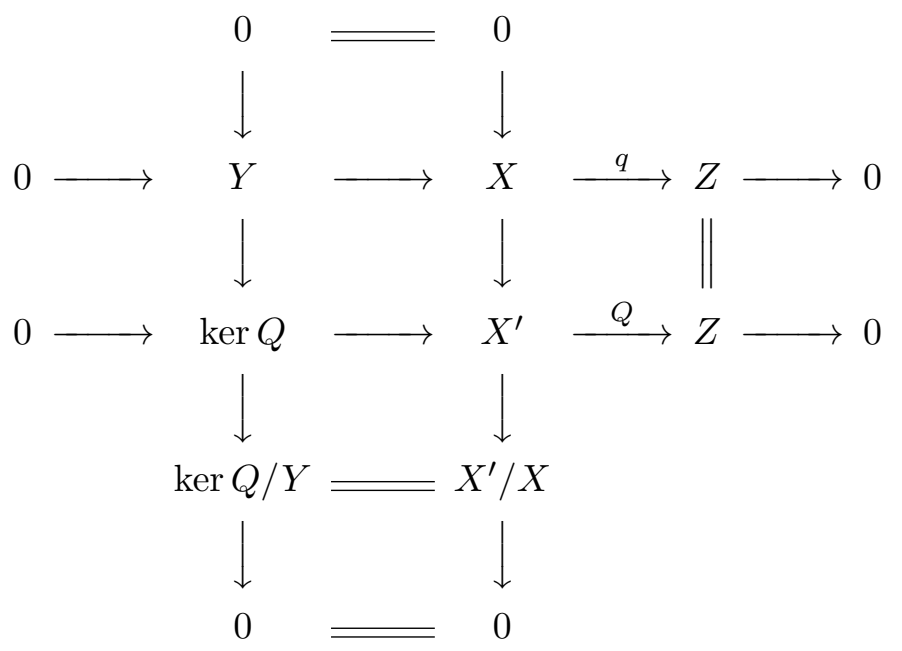

shows that $\operatorname{ker} Q$ is a superspace of $Y$ with $\operatorname{dens}(\operatorname{ker} Q / Y)<\aleph$. Let $A$ : $\operatorname{ker} Q \rightarrow Y^{\prime}$ be an extension of $\alpha$. Given $x^{\prime} \in X^{\prime}$ pick $x \in X$ so that $Q x^{\prime}=q x$ and define $B: X^{\prime} \rightarrow$ PO by means of

$$
B\left(x^{\prime}\right)=\left(A\left(x^{\prime}-x\right), x\right)+\Delta .
$$

To check it is well defined, observe that if $Q\left(x^{\prime}\right)=q(x)=q(w)$ then

$$
\left(A\left(x^{\prime}-x\right), x\right)-\left(A\left(x^{\prime}-w\right), w\right)=(A(w-x), x-w) \in \Delta
$$

since $x-w \in Y$. The operator $B$ is continuous since

$$
\begin{aligned}
\left\|B x^{\prime}\right\| & =\inf _{y \in Y}\left\|\left(A\left(x^{\prime}-x\right), x\right)-(A y,-y)\right\| \\
& =\inf _{y \in Y}\left\|\left(A\left(x^{\prime}-x-y\right), x+y\right)\right\| \\
& \leq \inf _{y \in Y}\left\|A\left(x^{\prime}-x-y\right)\right\|+\|x+y\| \\
& \leq\left\|A x^{\prime}\right\|+2\|q x\| \\
& \leq\|A\|\left\|x^{\prime}\right\|+2\left\|Q x^{\prime}\right\| \\
& \leq(\|A\|+2\|Q\|)\left\|x^{\prime}\right\| .
\end{aligned}
$$

Finally, $B$ is an extension of $\beta$ since when $x \in X$ one has

$$
B(x)=(A(x-x), x)+\Delta=(0, x)+\Delta=\beta(x) .
$$


We do not know however if the operator ideal $\mathcal{E}_{0}$ satisfies the $3 S_{+}$. Thus, by the result [13, Proposition 18] above mentioned, we get that the class $S p\left(\mathcal{E}_{0}(\aleph)\right)$ of $\aleph$-injective Banach spaces has the 3 -space property. This fact is well-known (see $[6,5]$ ). The homological argument can be found in [9]: every property having the form $\operatorname{Ext}(\cdot, X)=0 \operatorname{or} \operatorname{Ext}(X, \cdot)=0$ is a 3 -space property. A forerunner can be found in [15]. By Lemma 1 , the class $\mathcal{E}_{0}(\aleph)^{d}$ has property $3 S_{+}$, from where it follows also that $S p\left(\mathcal{E}_{0}\left(\aleph^{d}\right)\right.$ has the 3 -space property, something we already knew since it is a standard fact that if $S p(\mathcal{A})$ has the 3 -space property then also $S p\left(\mathcal{A}^{d}\right)$ has the 3 -space property. Moreover, to identify the class $S p\left(\mathcal{E}_{0}(\aleph)^{d}\right)$ is easy and, surprisingly, the class turns out to be independent of $\aleph$ :

Proposition 8. For every $\aleph, S p\left(\mathcal{E}_{0}(\aleph)^{d}\right)$ is the class of $\mathcal{L}_{1}$-spaces.

Proof. Recall [5] that $\aleph$-injective spaces are $\mathcal{L}_{\infty^{-}}$-spaces and that dual $\mathcal{L}_{\infty^{-}}$ spaces are injective. Thus, $X^{*}$ is $\aleph$-injective if and only if it is an $\mathcal{L}_{\infty}$-space, which occurs if and only if $X$ is an $\mathcal{L}_{1}$-space.

Curiously enough, if one defines the apparently dual classes $\mathcal{L}(\aleph)$ of operators that can be lifted to any superquotient having kernel with density character strictly lesser than $\aleph$ the identification of $\operatorname{Sp}(\mathcal{L}(\aleph))$ is not so simple. Indeed, observe that a Banach space in $S p(\mathcal{L}(\aleph))$ must be $\aleph$-projective, with the obvious meaning that $\operatorname{Ext}(X, Y)=0$ for every Banach space $Y$ with $\operatorname{dens}(Y)<\aleph$. It is clear that a separable separably projective must be $\ell_{1}$. It is also clear that an $\aleph$-projective space is such that any $\aleph$-projective space must be an $\mathcal{L}_{1}$-space with the Schur property (see [5] for details). There are however uncountably many non-mutually isomorphic $\mathcal{L}_{1}$-subspaces of $\ell_{1}$. It is likely that that the answer to the following question be positive.

QUESTION 1. Is a separably projective space projective? Equivalently [24, Theorem C.3.8], is a separably projective space isomorphic to $\ell_{1}(I)$ for some set $I$ ?

Turning to the main topic of this paper, observe that the case of the ideal $\mathcal{E}(\aleph)$ is quite different form that of $\mathcal{E}_{0}(\aleph)$ since, surprisingly, one has

Lemma 3. $\mathcal{E}(\aleph)$ does not enjoy either $3 S_{-}$or $3 S_{+}$.

Proof. Otherwise, the associated space class $S p\left(\mathcal{E}_{0}(\aleph)\right)$ of universally $\aleph-$ injective spaces would enjoy the 3 -space property, something that, under $\mathrm{CH}$, has been shown to be false in [7]. 


\section{REFERENCES}

[1] P. Aiena, M. González, A. Martínez-Abejón, Operator semigroups in Banach space theory, Boll. Unione Mat. Ital. Sez. B Artic. Ric. Mat. (8) $4(1)(2001), 157-205$.

[2] F. Albiac, N.J. Kalton, "Topics in Banach Space Theory", Graduate Texts in Math. 233, Springer, New York, 2006.

[3] T. Alvarez, M. González, Some examples of tauberian operators, Proc. Amer. Math. Soc. 111 (4) (1991), 1023-1027.

[4] K. Astala, H.-O. Tylli, Seminorms related to weak compactness and to tauberian operators, Math. Proc. Cambridge Phil. Soc. 107 (2) (1990), $365-375$.

[5] A. Avilés, F. Cabello Sánchez, J.M.F. Castillo, M. González, Y. Moreno, "Separably Injective Banach Spaces", Lecture Notes in Math. 2132, Springer, 2016.

[6] A. Avilés, F. Cabello Sánchez, J.M.F. Castillo, M. González, Y. Moreno, On separably injective Banach spaces, Adv. Math. 234 (2013), $192-216$.

[7] A. Avilés, F. Cabello Sánchez, J.M.F. Castillo, M. González, Y. Moreno, Corrigendum to "On separably injective Banach spaces [Adv. Math. 234 (2013), 192-216]", Adv. Math. 318 (2017), 737-747.

[8] S.F. Bellenot, The $J$-sum of Banach spaces, J. Funct. Anal. 48 (1) (1982), $95-106$.

[9] F. Cabello Sánchez, J.M.F. Castillo, The long homology sequence for quasi-Banach spaces, with applications, Positivity 8(4) (2004), $379-$ 394.

[10] F. Cabello Sánchez, J.M.F. Castillo, N.J. Kalton, Complex interpolation and twisted twisted Hilbert spaces, Pacific J. Math. $276(2)$ (2015), $287-307$.

[11] J.M.F. Castillo, On Banach spaces $X$ such that $L\left(L_{p}, X\right)=K\left(L_{p}, X\right)$, Extracta Math. 10 (1) (1995), 27-36.

[12] J.M.F. CAstillo, M. GonzÁlez, "Three-space Problems in Banach Space Theory", Lecture Notes in Math. 1667, Springer-Verlag, Berlin, 1997.

[13] J.M.F. Castillo, M. González, A. Martínez-Abejón, Classes of operators preserved by extension and lifting, J. Math. Anal. Appl. 462 (1) (2018), $471-482$.

[14] J.M.F. Castillo, M. Simões, J. SuÁrez, On a question of Pełczyński about the duality problem for weakly compact strictly singular operators, Bull. Pol. Acad. Sci. Math. 60 (1) (2012), $27-36$.

[15] P. Domański, " $\mathcal{L}_{p}$-spaces and Injective Locally Convex Spaces", Dissertationes Math. 298, 1990.

[16] P. Domański, Ideals of extendable and liftable operators, RACSAM, Rev. R. Acad. Cienc. Exactas Fís. Nat. Ser. A Mat. 97 (2) (2003), 229-241. 
[17] M. GonzÁlez, A. Martínez-Abejón, Ultrapowers and semi-Fredholm operators, Boll. Un. Mat. Ital. B (7) 11 (2) (1997), 415-433.

[18] M. GonzÁlez, A. Martínez-Abejón, "Tauberian Operators", Operator Theory: Advances and Applications 194, Birkhäuser Verlag, Basel, 2010.

[19] M. GonzÁlez, V. M. Onieva, Lifting results for sequences in Banach spaces, Math. Proc. Cambridge Philos. Soc. 105 (1) (1989), 117-121.

[20] M. GonzÁlez, V. M. Onieva, Characterizations of tauberian operators and other semigroups of operators, Proc. Amer. Math. Soc. 108 (2) (1990), $399-405$.

[21] M. González, E. Saksman, H.-O. Tylli, Representing non-weakly compact operators, Studia Math. 113 (3) (1995), 265-282.

[22] N. Kalton, A. Wilansky, Tauberian operators on Banach spaces, Proc. Amer. Math. Soc. 57 (2) (1976), 251-255.

[23] J. Lindenstrauss And L. Tzafriri, "Classical Banach spaces II, Function Spaces", Ergebnisse der Math. und ihrer Grenzgebiete 97, SpringerVerlag, Berlin-New York, 1979.

[24] A. Pietsch, "Operator Ideals", North-Holland Publishing Co., AmsterdamNew York, 1980.

[25] A. Pietsch, "History of Banach Spaces and Linear Operators", Birkhauser, Boston, MA, 2007.

[26] H. Rosenthal, On wide-(s) sequences and their applications to certain classes of operators, Pacific J. Math. 189 (2) (1999), 311-338.

[27] D.G. TACon, Generalized semi-Fredholm transformations, J. Austral. Math. Soc. 34 (1) (1983), $60-70$.

[28] Q. Zeng, Five short lemmas in Banach spaces, Carpathian J. Math. 32 (1) (2016), $131-140$.

[29] Q. Zeng, H. Zhong, Three-space theorem for semi-Fredholmness, Arch. Math. (Basel) 100 (1) (2013), 55-61. 
